\title{
Volksgesetzgebung oder Volksveto? Überlegungen zur institutionellen Ausgestaltung der Direktdemokratie in der Bundesrepublik
}

\author{
Frank Decker
}

Die Debatte um die Einführung, Erweiterung und Nutzung direktdemokratischer Beteiligungsformen in der Bundesrepublik hat einen neuen Höhepunkt erreicht. Dafür verantwortlich sind mehrere Ereignisse, deren Ein- bzw. Zusammentreffen zum Teil zufällig ist, zum Teil durchaus absehbar war. Die heftige Diskussion um das Buch von Thilo Sarrazin ${ }^{1}$, die eine tiefe Kluft zwischen der demoskopisch feststellbaren Volksmeinung und den Positionen der politischen Entscheidungsträger offenbart hat, und die Auseinandersetzung um die Wahl des Bundespräsidenten, bei der sich der Kandidat des Establishments gegen den mehrheitlichen Favoriten der Bevölkerung durchsetzte, gehören in die erste Kategorie - sie liefern Belege für die tatsächliche oder behauptete Krise der repräsentativen parteiendemokratischen Institutionen. In dieselbe Richtung weist der massive Protest gegen das Großprojekt eines Bahnhofneubaus in Stuttgart, der zudem von der konkreten Forderung nach einer Volksabstimmung begleitet wird.

Gleichzeitig bzw. parallel dazu haben die Bürger in zwei Bundesländern Bayern und Hamburg - mithilfe der direktdemokratischen Instrumente zentrale Gesetzesvorhaben der Regierenden ausgehebelt. Da beide Länder vergleichsweise niedrige Hürden für den Einsatz der Plebiszite vorsehen, musste mit einer solchen Situation früher oder später gerechnet werden. Besondere Brisanz barg der Konflikt in Hamburg, weil sich der Bürger(un)wille hier gegen diejenigen richtete, die sich zuvor für eine erleichterte Nutzung der direktdemokratischen Verfahren stark gemacht hatten. Dies hat bei den Befürwortern zu einer neuen Nachdenklichkeit geführt, ob man auf dem bisherigen Weg der Verfahrenserleichterungen weiterfahren kann oder soll. Zusätzliche Irritationen weckte der Ausgang der jüngsten Volksabstimmungen in den direktdemokratischen Musterländern Schweiz (Minarettinitiative) und Kalifornien (Staatshaushalt), die nicht gerade als Beleg einer höheren oder im Vergleich zu den Repräsentanten zumindest gleichwertigen Vernunft der Bürger taugen.

1 T. Sarrazin, Deutschland schafft sich ab. Wie wir unser Land aufs Spiel setzen, 2010. 
Die aktuelle Diskussion ist insofern von einer grundlegenden Ambivalenz gekennzeichnet. Auf der einen Seite mehren sich die Forderungen nach mehr direkter Demokratie, um Legitimationsdefizite der parlamentarischen Parteiendemokratie auszugleichen. ${ }^{2}$ Auf der anderen Seite wachsen die Zweifel an der bestehenden Form der „Volksgesetzgebung“, nachdem diese in einigen Bundesländern begonnen hat, den politischen Prozess massiv zu beeinflussen. Beide Positionen können sich, wie ich zeigen möchte, auf gewichtige Argumente stützen. Sie lassen sich zusammenführen, wenn man die Fixierung auf das Volksgesetzgebungsmodell aufgibt, die Befürworter und Gegner der direkten Demokratie in der Bundesrepublik eint. Wie dies geschehen kann und welche Konsequenzen für die Ausgestaltung der plebiszitären Verfahren sich daraus auf der Länderund Bundesebene ergeben, ist Gegenstand der nachfolgenden, thesenhaft vorgetragenen Überlegungen.

\section{Direktdemokratische und parlamentarische Repräsentation dürfen nicht an unterschiedlichen Prinzipien gemessen werden.}

Anders als die territoriale Herrschaftsgliederung, die Verfassungsgerichtsbarkeit oder das Wahlsystem gehören plebiszitäre Verfahren nicht zu den zwingend notwendigen, sondern zu den „optionalen“ Einrichtungen eines demokratischen Regierungssystems. Wer sie neu einführen will, muss deshalb den Nachweis führen, dass sie sich in dessen bestehende Strukturen gut einfügen und ihnen ein demokratischer Mehrwert innewohnt. Letzteres setzt voraus, dass die Plebiszite über ihre symbolische Funktion hinaus tatsächlich eine politische Wirkung entfalten. Handelt es sich bei der Direktdemokratie um ein in der Praxis nicht einlösbares Versprechen an die Bürger, würde nicht nur deren eigene Legitimation beschädigt, sondern die der gesamten parlamentarischen Parteiendemokratie.

Liegt die Beweislast von daher bei denjenigen, die das bestehende System verändern wollen, bedeutet das andererseits nicht, dass die neu einzuführenden plebiszitären Elemente an anderen, höheren Maßstäben gemessen werden dürfen als die vorhandenen repräsentativen Verfahren. Eine solche Tendenz ist auch in der wissenschaftlichen Debatte häufig beobachtbar. So wird z.B. mit Blick auf den Ausgang von Volksabstimmungen regelmäßig die Vernunftbegabung des Volkes zu einem Thema gemacht, die man gewählten Vertretern scheinbar $a$ priori zuschreibt. Dasselbe gilt für die niedrigen Beteiligungsraten, die die Legi- 
timität einer vom Volk getroffenen Mehrheitsentscheidung angeblich in Frage stellen, während eine vergleichbare Konsequenz aus der ebenfalls stark rückläufigen Beteiligung bei den regulären Wahlen eher nicht gezogen wird. Damit verbunden rufen auch die Einflussnahme von Lobbyisten und interessierten Abstimmungsminderheiten größeren Argwohn hervor, wenn sie im Kontext direktdemokratischer Verfahren stattfinden. Im Rahmen der herkömmlichen repräsentativen Demokratie hat man sich an solche Erscheinungen offensichtlich gewöhnt.

Das Problem beginnt bereits bei einem Begriffsverständnis, das direkte (plebiszitäre) und repräsentative Demokratie als Gegensatz auffasst. Versteht man unter „Repräsentation“ die Ausübung von Herrschaft im Auftrag oder Namen des Volkes, mithin das stellvertretende Entscheiden, erscheint das nicht unbedingt plausibel. Auch das Volk trifft ja, wenn es zum Plebiszit aufgerufen ist, seine Entscheidung immer stellvertretend für jene, die daran nicht teilnehmen wollen, dürfen oder können. Zu den beiden letztgenannten Gruppen gehören z.B. Kinder oder die künftigen Generationen. Die plebiszitäre Demokratie stellt insofern nur eine Variante der repräsentativen Demokratie dar, bei der die Gesetzgebung nicht den gewählten Vertretern, sondern den Wählern selbst obliegt, die dadurch in den Status einer Verfassungsinstitution hineinwachsen: Die Trennlinie verläuft zwischen parlamentarischer und plebiszitärer Repräsentation, nicht zwischen repräsentativer und plebiszitärer Demokratie. ${ }^{3}$

Dies vorausgesetzt, unterliegen parlamentarische und plebiszitäre Repräsentation denselben Kriterien der Input- und Output-Legitimation, die Lincoln in seiner Definition des ,government by the people and for the people“ formuliert hat. Die Input-Legitimation entspricht der ursprünglichen Idee der Volkssouveränität, wonach die Herrschaft vom Volkswillen ausgehen bzw. von diesem getragen sein muss. Konkretisiert wird sie in der Forderung nach Inklusion, das heißt der Beteiligung der Bürger an den sie betreffenden Entscheidungsprozessen. Diese Forderung kann weit oder eng ausgelegt werden. In der weiten Auslegung der partizipatorischen Demokratietheorie zielt sie auf eine möglichst umfassende und intensive Teilhabe aller in sämtlichen Phasen der Willens- und Entscheidungsbildung, die vom Gleichheitsgedanken ausgeht und über den staatlichen Bereich hinaus potenziell die ganze Gesellschaft erfasst. ${ }^{4}$ In der engeren Auslegung basiert sie auf der Kontroll- und Sanktionsmacht, die den Bürgern über die Wahlen zuwächst. Die Output-Legitimation orientiert sich dagegen weniger am

3 W. Steffani, Das magische Dreieck demokratischer Repräsentation: Volk, Wähler und Abgeordnete, in: ZParl 30 (1999), 772 (774).

4 M.G. Schmidt, Demokratietheorien. Eine Einführung, 4. Auflage 2008, S. 236 ff. 
Zustandekommen als an den Ergebnissen des politischen Prozesses. Sie gründet auf möglichst effektiven und effizienten Problemlösungen, das heißt auf der Fähigkeit zum ,guten Regieren“. Ihr Hauptaugenmerk liegt auf der Steigerung der staatlichen Handlungskapazität im Interesse einer aktiven, dem Gemeinwohl verpflichteten Politik.

Input- und Output-Legitimation können im Einzelfall in Widerstreit geraten, bleiben aber im Kern aufeinander bezogen. ${ }^{5}$ Denn einerseits herrscht über das, was das Gemeinwohl gebietet, politischer Streit, da die Entscheidungsbeteiligten unterschiedliche Meinungen vertreten und Ziele verfolgen. Diese müssen folglich zu einem Ausgleich gebracht werden. Andererseits nützen inhaltlich richtige Entscheidungen wenig, wenn sie nicht zugleich bei den Adressaten auf Akzeptanz stoßen. Auch das kann nur über einen entsprechenden Input gewährleistet werden. Mangelt es an der Folgebereitschaft, verfehlen die Entscheidungen entweder ihre Wirkung. Oder sie rufen Protest und Widerstand hervor, der sich im schlimmsten Fall zu einem anhaltenden Loyalitätsentzug gegenüber der Politik und dem politischen System auswächst.

Die Skepsis gegenüber plebiszitären Elementen gründete lange Zeit auf deren vermeintlicher Unterlegenheit unter Output-Gesichtspunkten. Direktdemokratische Verfahren tendierten nach dieser Auffassung dazu, die Politik zu „entrationalisieren“, weil ihnen die deliberativen Eigenschaften der repräsentativen Verfahren fehlten. Nicht nur, dass dem Volk die Kompetenz abgehe, die in der Regel komplexen Entscheidungsmaterien zu überblicken. Es sei auch in seiner grundsätzlichen Orientierung weniger dem Gemeinwohl als den eigenen Interessen verpflichtet und unterliege dabei starken Stimmungsschwankungen. Im Ergebnis führe das zu einer geringeren Entscheidungsqualität.

Der Glaube an die angeblich höhere Vernunft der parlamentarischen Repräsentation entfaltet zwar immer noch eine autosuggestive Wirkung. Seine empirische Basis ist im Laufe der Zeit allerdings schwächer geworden. Einerseits konnte man für eine diesbezügliche Unterlegenheit der Plebiszite keine Belege beibringen. Untersuchungen aus der Schweiz und den US-Einzelstaaten zeigten im Gegenteil, dass die Bürger in bestimmten Politikbereichen sogar sachgerechtere Entscheidungen trafen als die Repräsentanten. ${ }^{6}$ Andererseits ließ sich nicht ignorieren, dass die repräsentativen Institutionen selbst von plebiszitären Tendenzen in Beschlag genommen wurden, die die Qualität ihrer Entscheidungen beein-

5 H. Abromeit, Wozu braucht man Demokratietheorie? Die postnationale Herausforderung der Demokratietheorie, 2002, S. 15 ff.

6 M. Freitag/U. Wagschal (Hrsg.), Direkte Demokratie. Bestandsaufnahmen und Wirkungen im internationalen Vergleich, 2007. 
trächtigten. In der Forschung hat sich deshalb die Meinung durchgesetzt, dass eine klare Präferenz für oder gegen die direkte Demokratie von der Outputseite her nicht (mehr) begründet werden kann.

Anders stellt sich die „Gefechtslage“ auf der Inputseite dar. Hier wird den Plebisziten von deren Befürwortern zumeist eine höherwertige Legitimation zugeschrieben, da der Bürger ,nun mal bei direktdemokratischen Verfahren mehr Chancen [habe], auf den Inhalt der Entscheidungen einzuwirken als bei Entscheidungen durch Repräsentanten“7 . Gegen diese Position ist nur schwer anzukommen, obwohl sie aus normativer Sicht ebenso wenig überzeugt wie die Präferenz für die parlamentarische Repräsentation auf der Outputseite. Ihre Schwäche liegt darin, dass sie Bürgermitwirkung irrtümlich mit „Responsivität“ gleichsetzt. Aus Sicht der an einer Abstimmung tatsächlich Teilnehmenden mag beides Hand in Hand gehen. Ob die direkte Demokratie auch die Präferenzen der übrigen Bürger besser oder genauso gut abbilden kann wie eine repräsentative Körperschaft, müsste aber empirisch erst bewiesen werden. Die Literatur liefert hier$\mathrm{zu}$,ein ebenso facettenreiches wie disparates Bild“8

Ein demokratischer Mehrwert der plebiszitären Verfahren kann demnach nicht von vornherein unterstellt werden. Wenn er ihnen dennoch zugebilligt wird, dürfte das vor allem damit zusammenhängen, dass es sich bei den vom Volk beschlossenen oder verworfenen Gesetzen überwiegend um solche handelt, die als besonders wichtig empfunden werden und den Charakter von Richtungsentscheidungen gewinnen. Das Gros der Gesetzgebungsarbeit verbleibt demgegenüber auch in den Staaten mit einer breit ausgebauten Direktdemokratie in den Händen der Repräsentativkörperschaften. Der Unterschied zeigt sich z.B. bei der verfassungsgerichtlichen Kontrolle. Diese erstreckt sich im Prinzip gleichermaßen auf volksbeschlossene und Parlamentsgesetze. Handelt es jedoch um Richtungsentscheidungen, denen eine größeren Legitimität zugeschrieben wird, wenn sie direktdemokratisch zustande kommen, dann würde das Verfassungsgericht einen solchen Volksentscheid nicht einfach kassieren können, ohne seine eigene Legitimation auszuhöhlen. ${ }^{9}$ Denselben Unterschied erkennen die US-amerikani-

7 H.H. v. Arnim, Vom schönen Schein der Demokratie. Politik ohne Verantwortung - am Volk vorbei, 2000, S. $191 \mathrm{ff}$.

8 F. Grotz, Direkte Demokratie in Europa. Erträge, Probleme und Perspektiven der vergleichenden Forschung, in: PVS 50 (2009), 286 (298).

9 Die Befürworter einer plebiszitären Ergänzung des Grundgesetzes versuchen dieses Problem durch eine verfassungsgerichtliche Ex ante-Kontrolle zu umgehen. So hat der von der rot-grünen Koalition 2002 eingebrachte Entwurf vorgesehen, dass bei begründeten Zweifeln an der Verfassungsmäßigkeit eines vom Volk begehrten Gesetzes schon vorab eine Entscheidung aus Karlsruhe einzuholen sei. Die Prüfung erfolgt auf Antrag, nach- 
schen Einzelstaatsverfassungen an, wenn sie volksbeschlossene Gesetze vom Veto des Gouverneurs ausnehmen.

Die Behauptung einer normativen Höherwertigkeit der Direktdemokratie ist auch deshalb prekär, weil sie mit Blick auf deren Möglichkeiten völlig unrealistische Erwartungen weckt. Indem sie die politischen Eliten anhalten, ihr Tun und Unterlassen vor der Wählerschaft sorgfältiger zu begründen, könnten die Plebiszite gewiss einen Beitrag leisten, um die Bürger wieder näher an die repräsentativen Institutionen heranzuführen. Die Vorstellung, dass damit das Legitimationsdefizit der Parteiendemokratie schon zu beheben sei, mutet jedoch naiv an. Deren Repräsentationsschwäche ist ja nicht in erster Linie ein Problem mangelnder kommunikativer Vermittlung. Sie wurzelt vielmehr in den sozialen Gegensätzen einer zunehmend auseinanderdriftenden Gesellschaft, die das materielle Substrat der Gleichheitsidee untergraben, auf der die Demokratie beruht. Die empirischen Befunde zeigen, dass es gerade die marginalisierten Wählerschichten sind, die das Vertrauen in die Politik verloren haben und sich von ihr dauerhaft abwenden. Wenn diese Wähler heute bereits die Wahlen schmähen, die immer noch die gleichheitsfreundlichste, weil niedrigstschwellige Form der Beteiligung darstellen, dann werden sie sich auch durch die höherschwelligen Partizipationsangebote der Direktdemokratie nicht in das System zurückholen lassen. ${ }^{10}$

\section{Ein Gesetzgebungsrecht des Volkes passt nicht zur gewaltenfusionierenden} Logik des parlamentarischen Systems

In der Literatur findet man häufig die Feststellung, repräsentative und direkte Demokratie stünden in einem komplementären Verhältnis zueinander. Die repräsentative Demokratie ist danach das ursprünglichere oder Regelprinzip; sie geht den plebiszitären Institutionen voraus und wird durch diese beeinflusst bzw. um einen alternativen Pfad der demokratischen Willens- und Entscheidungsbildung erweitert. Das Regel-Ausnahme-Verhältnis schlägt sich zum einen darin nieder, dass Volksabstimmungen nur selten vorkommen; selbst in den Vorreiterländern der direkten Demokratie ist ihre Zahl im Vergleich zu den Regierungsentscheidungen und parlamentarisch beschlossenen Gesetzen verschwindend gering.

dem das Volksbegehren eingereicht ist. Anrufungsbefugt wären - wie bei der abstrakten Normenkontrolle üblich - die Bundesregierung, eine Landesregierung oder ein Drittel der Mitglieder des Bundestages.

10 A. Schäfer, Die Folgen sozialer Ungleichheit für die Demokratie in Westeuropa, in: ZvglPolW 4 (2010), $131 \mathrm{ff}$. 
Zum anderen wirken die Plebiszite, auch wo sie als Initiativrechte ausgestaltet sind, eher nachträglich oder korrigierend, stellen sie also eine Reaktion auf die Politik der repräsentativen Institutionen dar, denen die aktive Gestaltung der Regierungs- und Gesetzgebungstätigkeit zukommt.

In beiderlei Hinsicht können die direktdemokratischen Verfahren allerdings eine Wirkung ausüben, die über eine bloß „,ergänzende“ Funktion hinausweist. Trifft das Volk die grundlegenden Richtungsentscheidungen selbst und werden die repräsentativen Institutionen in ihrem eigenen Entscheidungsverhalten durch die direktdemokratischen Verfahren präjudiziert, führt die Rede vom Regel-Ausnahme-Verhältnis in die Irre. Das naheliegende Beispiel ist die Schweiz, wo die Volksrechte nicht nur subsidiär wirken, sondern die Funktionslogik des gesamten Regierungssystems prägen. Die Konkordanzregierung, in die alle wichtigen Parteien des Landes einbezogen sind, und die direktdemokratischen Institutionen bilden hier Seiten derselben Medaille.

Ihr reaktiver, nachträglicher Charakter macht die Plebiszite zu einem (potenziellen) Mittel der Opposition; durch sie nimmt das Volk die Rolle eines „Vetospielers“ im Regierungsprozess ein. In der klassischen rechts- und politikwissenschaftlichen Terminologie könnte man auch von einer zusätzlichen Spielart der Gewaltenteilung sprechen, die in das bestehende System von Exekutive, Legislative und Judikative integriert wird. Gilt diese Feststellung ganz allgemein, so unterscheidet sich der oppositionelle Charakter der Direktdemokratie je nach Ausgestaltung erheblich. Am schwächsten ausgeprägt ist er dort, wo die Regierung oder das Parlament dem Volk ein Gesetz (oder eine sonstige Angelegenheit) nach eigenem Ermessen zur Entscheidung vorlegen (,einfaches“ Referendum). Die stärkste Ausprägung erfährt er, wenn das Volk selber das Recht hat, über ein bereits beschlossenes Gesetz einen Volksentscheid zu verlangen. Diese Form der „Vetoinitiative“ ist in der Schweiz unter dem Begriff „fakultatives Referendum“ geläufig. Von ihr zu unterscheiden ist die ,positive“ Gesetzesinitiative, die in der Bundesrepublik unter dem Begriff „Volksgesetzgebung“ firmiert. Diese geht noch einen Schritt weiter, indem sie dem Volk auch die Möglichkeit gibt, Gesetze vorzuschlagen. Hier können die Bürger also selber festlegen, über welche Inhalte am Ende abgestimmt wird.

Die Einordnung der ,positiven“ Initiative als reaktives Instrument erscheint im Vergleich zur ,negativen“ Vetoinitiative auf den ersten Blick wenig überzeugend. Der in der Schweiz übliche Sprachgebrauch, wonach die negative Initiative als „Bremse“ und die positive Initiative als „Gaspedal“ im Regierungsprozess 
fungiert, trägt dem Rechnung. ${ }^{11}$ Er übersieht aber, dass auch die eigenen Initiativen des Volkes stets eine Reaktion auf das Tun oder Unterlassen der repräsentativen Institutionen darstellen. Würden Regierung und Gesetzgeber immer im Interesse und zur Zufriedenheit der Bürger handeln, wäre es ja nicht notwendig, das „Gaspedal“ zu betätigen. Hinzu kommt, dass das Volk die Funktion eines Gesetzgebers schon in einem technischen Sinne kaum wahrnehmen kann, weil ihm der dazu nötige administrative Unterbau fehlt. Auf der nationalen Ebene erstreckt sich die Initiative in der Schweiz deshalb nur auf Verfassungsänderungen; die kompliziertere „einfache“ Gesetzgebung obliegt ausschließlich Parlament und Regierung.

Noch wesentlich evidenter ist der reaktive Charakter der Direktdemokratie in der Bundesrepublik. Weil die deutschen Länderverfassungen fast nur das ,positive" Instrument der Volksgesetzgebung kennen, übernimmt diese in der Praxis zugleich den Part der nicht vorgesehenen Vetoinitiative, indem sich die positiven Initiativen - wie soeben wieder in Bayern und Hamburg - explizit gegen zuvor verabschiedete oder geplante Gesetzesvorhaben wenden. Wertet man die Volksbegehren, die im Zeitraum von 1947 bis 2010 in den Ländern insgesamt stattgefunden haben, unter diesem Gesichtspunkt aus, handelt es sich in zwei Dritteln der Fälle de facto um Vetoinitiativen. Die Frage ist daher berechtigt, warum der Verfassunggeber die Möglichkeit eines solchen Gesetzesvetos nicht von vornherein eröffnet hat (und ob er das in Zukunft tun sollte).

Die Charaktersierung der Direktdemokratie als Vetoinstrument lässt sich auch am - in der Bundesrepublik zuletzt stark diskutierten - Problem der Verbindlichkeit der volksbeschlossenen Gesetze festmachen. ${ }^{12}$ Verfassungsrechtlich sind Volks- und Parlamentsgesetze prinzipiell gleichrangig. Damit wären sie zugleich wechselseitig aufhebungsfähig: So wie das Volk die Möglichkeit hat, ein Parlamentsgesetz einem Referendum zu unterwerfen oder durch eine spätere (positive) Gesetzesinitiative zu korrigieren, so muss auch das Parlament ein vom Volk beschlossenes Gesetz wieder rückgängig machen können. Verfassungspolitisch

11 W. Linder, Schweizerische Demokratie. Institutionen - Prozesse - Perspektiven, 2. Aufl. 2005, S. 264 ff. In der Unterscheidung schwingt auch die Unterstellung mit, dass die Bremse eher konservative (oder ideologisch rechte) und das Gaspedal eher progressive (oder ideologisch linke) Politiken befördere. Wie problematisch eine solche Gleichsetzung ist, zeigt der Erfolg der positiven (Verfassungs)initiativen für ein Minarettverbot und die Ausweisung krimineller Ausländer in der Schweiz, die beide von der rechtspopulistischen SVP lanciert wurden.

12 O. Jung, Volksgesetze und parlamentarische Konterlegislatur, in: K.H. Schrenk/M. Soldner (Hrsg.), Analyse demokratischer Regierungssysteme. Festschrift für Wolfgang Ismayr zum 65. Geburtstag, 2010, S. 427 ff. 
besteht eine solche Symmetrie jedoch nicht. Während die Korrektur eines Parlamentsgesetzes durch das Volk im Konzept der Direktdemokratie ausdrücklich intendiert ist, würde die Direktdemokratie ad absurdum geführt, wenn das Parlament einen Volksentscheid schon am nächsten Tage wieder aufhöbe. Volksbeschlossene Gesetze erfordern deshalb einen höheren Bestandsschutz als Parlamentsgesetze. Eine Lösung könnte z.B. darin liegen, dass man bestimmte Fristen vorsieht, innerhalb derer der parlamentarische Gesetzgeber auf ein plebiszitär zustande gekommenes Gesetz nicht zugreifen darf. Oder man macht die Aufhebung oder Korrektur eines solchen Gesetzes von einem neuerlichen Volksvotum abhängig bzw. unterwirft sie der Möglichkeit einer Vetoinitiative, wie es die in diesem Punkt geänderte Hamburgische Verfassung in Art 50 Abs. 4 und 4a jetzt vorsieht. ${ }^{13}$

Der Konflikt zwischen dem parlamentarischen und Volksgesetzgeber, den man in Hamburg auf diese Weise zu befrieden versucht hat, wirft die Frage auf, ob die plebiszitären Verfahren sich in das vorhandene parlamentarische System überhaupt sinnvoll einbetten lassen. Der europaweite Vergleich gibt hier zu denken. Während die Möglichkeit eines von oben anzuberaumenden Referendums in unterschiedlichen Varianten fast überall gegeben ist, gilt dies nämlich nicht für die Volksinitiative! Lässt man Zwergstaaten wie Liechtenstein oder San Marino außer Betracht, räumen in Europa lediglich fünf parlamentarische Demokratien - Italien, Litauen, die Slowakei, Slowenien und Ungarn - den Bürgern das Recht ein, ein vom Parlament beschlossenes Gesetz mittels Vetoinitiative aufzuheben. Drei davon - Litauen, die Slowakei und Ungarn - gestehen dem Volk darüber hinaus ein positives Gesetzgebungsrecht zu, das sich allerdings nicht auf alle Materien erstreckt. Von Italien abgesehen ist die Volksinitiative auf der nationalen Ebene also nur in einigen mittel- und osteuropäischen Ländern verbreitet, wo man sie nach dem Systemumbruch 1989 aus einem demokratischen Überschwang heraus eingeführt hat. ${ }^{14}$

Unter den präsidentiellen Systemen ist das Bild gemischter. Während sich die US-amerikanische Bundesverfassung bis heute in plebiszitärer Enthaltsamkeit

13 Die Neuregelung kam auf Druck eines von „Mehr Demokratie“ lancierten Volksbegehrens zustande, das neben anderen Verbesserungen auch eine größere Verbindlichkeit der Volksentscheide anstrebte. Dem waren mehrere Fälle vorausgegangen, in denen der Senat bzw. die Bürgerschaft der Hansestadt Volksbeschlüsse missachtet oder gegen den Willen der Initiatoren korrigiert hatte. Vgl. F. Decker, Parlamentarische Demokratie versus Volksgesetzgebung. Der Streit um ein neues Wahlrecht in Hamburg, in: ZParl 38 (2007), $118 \mathrm{ff}$.

14 B. Ewert, Potentiale der direkten Demokratie in Litauen, Slowenien und Ungarn unter besonderer Berücksichtigung der politischen Kultur, 2007. 
übt, ist die Initiative - in Kombination mit dem Referendum - mittlerweile in den meisten Staaten Lateinamerikas geläufig. ${ }^{15}$ Auf der anderen Seite sehen in den USA immerhin rund die Hälfte der Einzelstaaten zum Teil sehr weitreichende Initiativrechte vor. ${ }^{16}$ Dasselbe gilt für die präsidentiell (auf der gliedstaatlichen Ebene) bzw. „quasi-präsidentiell“ (auf der nationalen Ebene) verfasste Schweiz - das Land mit der am stärksten ausgebauten Direktdemokratie in der Welt überhaupt. Die Schweiz kennt die Initiative auf der nationalen Ebene bis heute zwar nur als Verfassungsinitiative; die direktdemokratischen Verfahren konzentrieren sich hier - wie gesehen - nicht in der Volksgesetzgebung, sondern im fakultativen Referendum. Unter dem Strich bleibt damit aber das Ergebnis, dass beide Formen - die positive wie die negative Initiative - in einem parlamentarischen Regierungssystem offenbar schlechter aufgehoben sind als in einem präsidentiellen.

Der Grund dafür liegt in der unterschiedlichen Struktur und Funktionsweise der beiden Regierungstypen. ${ }^{17}$ Der Präsidentialismus ist ein System der Gewaltentrennung. Präsident und Parlament werden unabhängig voneinander bestellt und können sich in ihrem wechselseitigen Bestand nichts anhaben. Die Regierungsmacht ist zwischen beiden Organen geteilt, die im Gesetzgebungsprozess gleichermaßen über pro-aktive und reaktive Kompetenzen verfügen, auf deren Basis sie die Gesetzesvorhaben untereinander aushandeln. Opposition wird dabei fallweise ausgeübt; es gibt keine dauerhaft festgefügten Koalitionen. Tritt das Volk als weiterer Vetospieler hinzu, wird die Funktionslogik dieses Systems nicht prinzipiell gestört, auch wenn die Komplexität des Verhandlungsprozesses weiter zunimmt. Die plebiszitären Verfahren können sogar eine ausgesprochen nützliche Rolle spielen, wenn sie dazu beitragen, Blockaden zwischen Präsident und Parlament aufzulösen - diese gehören bekanntlich zu den Hauptschwächen des Präsidentialismus.

Bei der parlamentarischen Regierungsform handelt es sich demgegenüber um ein System der Gewaltenfusion. Die Regierung geht aus der Mehrheit des Parlaments hervor, die sie politisch trägt und mit ihr eine Einheit bildet. Zwischen regierender Mehrheit und Opposition besteht eine klar festgelegte Aufgabenteilung: Der Regierung gebührt das Monopol der politischen Gestaltung, während

$15 S$. Rinke/K. Stüwe, Politische Systeme Amerikas: Ein Vergleich, in: K. Stüwe/S. Rinke (Hrsg.), Die politischen Systeme in Nord- und Lateinamerika, 2008, S. 9 ff.

16 S. Moeckli, Direkte Demokratie in den Gliedstaaten der USA, in: Freitag/Wagschal, Demokratie (Fn. 6), S. 19 ff.

17 F. Decker, Ist die Parlamentarismus-Präsidentialismus-Dichotomie überholt? Zugleich eine Replik auf Steffen Kailitz, in: ZPol 19 (2009), 169 ff. 
die Opposition als parlamentarische Minderheit ganz auf ihre Kontroll- und Alternativfunktion zurückgeworfen bleibt, die sie mit dem Ziel wahrnimmt, die Regierung nach der kommenden Wahl abzulösen. Ein plebiszitäres Vetorecht würde dieses Prinzip unterlaufen. Mit seiner Hilfe könnte die Opposition von der Regierungsmehrheit beschlossene Gesetze ja schon im Vorfeld einer Wahl zu Fall bringen. Es entstünde also eine Konkurrenz von parlamentarischem Mehrheits- und Volkswillen, die dem Konzept der Gewaltenfusion widerspricht. Von daher lässt sich gut nachvollziehen, warum die alten parlamentarischen Demokratien (mit Ausnahme Italiens) sämtlich darauf verzichtet haben, die Initiative in ihre Verfassungen einzubauen.

Die Fixierung der deutschen Verfassunggeber auf die Volksgesetzgebung wirkt vor diesem Hintergrund befremdlich. Sie ist das Paradebeispiel einer „pfadabhängigen“ Entwicklung. Die Ursprünge des plebiszitären Gesetzgebungsrechts in Deutschland reichen bis in die Mitte des 19. Jahrhunderts zurück. Sie gründen vor allem auf den Ideen Moritz Rittinghausens - eines sozialistischen Politikers, der heute praktisch in Vergessenheit geraten ist. ${ }^{18}$ Rittinghausen konzipierte die Volksinitiative damals als Alternative zum monarchisch-konstitutionellen Repräsentativsystem, das der oppositionellen Arbeiterbewegung eine faire Mitwirkungsmöglichkeit am politischen Prozess versagte. Das Modell datiert also aus einer Zeit, in der in Deutschland nicht nur die Durchsetzung der parlamentarischen Regierungsform, sondern auch die Demokratisierung des Parlamentarismus in weiter Ferne lagen. Mit zunehmender Integration der Sozialdemokraten in das System verloren diese das Interesse an einer Fortentwicklung der direktdemokratischen Ansätze, an denen sie offiziell aber festhielten. Als sich nach dem Untergang des Kaiserreiches die Möglichkeit abzeichnete, plebiszitäre Elemente in die neu zu schaffende republikanische Verfassung einzubringen, griff man deshalb wie selbstverständlich auf die Initiative zurück. Eine wichtige Rolle spielte dabei auch die Rezeption der direktdemokratischen Verfahren in der Schweiz und in den US-amerikanischen Einzelstaaten, die sich am selben Modell orientierten. Dass die Initiative dort in ein ganz anderes, nämlich präsidentiell verfasstes Regierungssystem eingebettet war, trat den Verfassunggebern 1919/20 nicht ins Bewusstsein.

Die Defizite der Weimarer Verfassung und der Missbrauch der Volksabstimmungen durch die Nationalsozialisten führten auch nach 1945 nicht zu einer

18 R. Schiffers, Elemente direkter Demokratie im Weimarer Regierungssystem, 1971, S. 17 ff. 
grundsätzlichen Neubewertung der Plebiszite. ${ }^{19}$ Sämtliche Länderverfassungen, die 1946 und 1947, also vor dem Grundgesetz, in Kraft traten, sahen ein Gesetzgebungsrecht des Volkes vor. Dass die Bundesverfassung später auf den Weg einer strikt repräsentativ angelegten Demokratie gebracht wurde, hatte weniger mit den angeblich negativen Erfahrungen von Weimar zu tun als mit der veränderten politischen Großwetterlage im aufziehenden Kalten Krieg, die den Verfassunggeber davor zurückschrecken ließ, die potenziell „unberechenbaren“ Plebiszite einzuführen. ${ }^{20}$ Da sich das parlamentarische System der zweiten deutschen Republik - anders als sein Vorläufer - als Erfolgsgeschichte entpuppte, bestand lange Zeit keine Veranlassung, diese Skepsis abzulegen. Erst in den achtziger Jahren, als die Funktionsschwächen des repräsentativen Prinzips immer offener zutage traten, erwachte die Direktdemokratie aus ihrem Dornröschenschlaf. Deren Gleichsetzung mit der Volksgesetzgebung sollte von da an nicht nur die konkreten Reformprozesse in den Ländern in eine problematische Richtung lenken, sondern auch die Debatte um eine mögliche Einführung plebiszitärer Elemente auf Bundesebene.

\section{Die Option für die potenziell weitreichendste Form der Direktdemokratie bedingt deren restriktive Ausgestaltung und Handhabung in der Praxis.}

Die Probleme der systemischen Integration werden durch die konkrete verfassungsrechtliche Ausgestaltung der Volksgesetzgebung in den Ländern belegt, deren Einführung bzw. Ausbau mit einem weitgehenden Verzicht auf andere plebiszitäre Beteiligungsformen einhergeht. ${ }^{21}$ Der Verfassunggeber hat sich zwar für die vermeintlich großzügigste Variante der Direktdemokratie entschieden; er schränkt diese aber in der Umsetzung so stark ein, dass den Volksrechten im realen Verfassungsleben nur eine geringe Rolle zukommt. ${ }^{22}$ Beides hängt unmittel-

19 H.-J. Wiegand, Direktdemokratische Elemente in der deutschen Verfassungsgeschichte, 2006, S. $181 \mathrm{ff}$.

20 O. Jung, Kein Volksentscheid im Kalten Krieg! Zum Konzept einer plebiszitären Quarantäne für die junge Bundesrepublik 1948/49, in: APuZ B 45/1992, 16 ff.

$21 J$. Rux, Direkte Demokratie in Deutschland. Rechtsgrundlagen und Rechtswirklichkeit der unmittelbaren Demokratie in der Bundesrepublik Deutschland und ihren Ländern, 2008, S. $333 \mathrm{ff}$.

22 Die Gesamtzahl der im Zeitraum 1947 bis 2008 eingeleiteten Volksbegehren beläuft sich auf 61, von denen wiederum mehr als die Hälfte (34) in lediglich drei Ländern - Bayern, Brandenburg und Hamburg - stattfanden. Baden-Württemberg, Mecklenburg-Vorpommern und das Saarland verzeichnen bislang kein einziges Volksbegehren, Hessen und 
bar miteinander zusammen. Nicht obwohl, sondern gerade weil man den Bürgern die theoretisch weitreichendsten Mitwirkungsmöglichkeiten eröffnet, sieht man sich gezwungen, diese in der Praxis sogleich wieder zu entwerten! Bei einer zu großen Anwenderfreundlichkeit scheint der Verfassunggeber also gerade jene Konflikte zwischen parlamentarischem und Volksgesetzgeber zu befürchten, die die potenzielle Systemunverträglichkeit des plebiszitären Instruments ausmachen.

Die verfassungsrechtlichen Einschränkungen der Volksgesetzgebung lassen sich in mehrere Gruppen einteilen. Am bedeutsamsten sind die Ausschlussgegenstände, also die Themen, über die kein Volksgesetzgebungsverfahren stattfinden darf. Diese entziehen im Rahmen der sogenannten Finanztrias auch finanzwirksame Gesetze überwiegend dem plebiszitären Zugriff. Dasselbe gilt für Verwaltungsentscheidungen und den Nachvollzug von Bundesgesetzen, obwohl im deutschen Föderalismus gerade hier die Hauptaufgabe der Länder liegt. Darüber hinaus wird die Anwendbarkeit durch die in den verschiedenen Stadien des Verfahrens zu erfüllenden Quoren erschwert. Während die verfassungsrechtlichen Regelungen im Bereich der Ausschlussgegenstände eng beieinanderliegen, bestehen hier zwischen den Ländern sehr große Unterschiede, die sich in der Nutzungshäufigkeit und Erfolgswahrscheinlichkeit der Plebiszite niederschlagen. ${ }^{23}$ Weitere Restriktionen ergeben sich aus den einzuhaltenden Fristen, den Regeln der Kostenübernahme und der Terminierung des Volksentscheids (parallel oder nicht parallel zu einer regulären Wahl). Letzteres hat in Hamburg z.B. zur Einführung einer differenzierten Quorenlösung geführt. ${ }^{24}$ Last but not least ist das bereits angesprochene Problem der Verbindlichkeit zu nennen, das bisher ebenfalls nur in Hamburg eine befriedigende Antwort gefunden hat $^{25}$.

Ohne bei der schier unendlichen Regelungsvielfalt weiter ins Detail zu gehen, kann man den verfassungsrechtlichen Ist-Zustand der Direktdemokratie in den deutschen Länderverfassungen in drei Feststellungen zusammenfassen. Erstens bleiben die Anwendungsbedingungen in der Summe so restriktiv, dass die plebiszitären Elemente in der Verfassungspraxis ein Schattendasein fristen und eine

Rheinland-Pfalz, wo das Instrument schon seit 1946 bzw. 1947 besteht, nur jeweils eines. Vgl. Mehr Demokratie, Volksbegehrensbericht 2008, 2009.

23 C. Eder/R. Magin, Direkte Demokratie, in: M. Freitag/A. Vatter (Hrsg.), Die Demokratien der Bundesländer, 2008, S. 257 ff.

A. v. Arnauld, „Refolution“ an der Elbe: Hamburgs neue direkte Demokratie - Die Verfassungsänderungen der Jahre 2008 und 2009 im Kontext, in: L.P. Feld u.a. (Hrsg.), Jahrbuch für direkte Demokratie 2009, 2010, S. 90 (111 ff.).

$25 \mathrm{Zu}$ einer anderen Lösung in Bremen siehe den Beitrag von D. Schefold in diesem Band (unten S. 134 ff.). 
nachhaltige, systemverändernde Wirkung von ihnen nicht ausgeht. Die benutzerfreundlichsten Länder in der Bundesrepublik bewegen sich ungefähr auf demselben Niveau wie die benutzerunfreundlichsten Schweizer Kantone oder USBundesstaaten! Zweitens bestehen zwischen den Ländern in den Anwendungsbedingungen enorme Unterschiede, die sich in der tatsächlichen Nutzung der Verfahren widerspiegeln. In keinem anderen Bereich der Länderregierungssysteme ist die institutionelle Bandbreite heute so groß wie hier. Und drittens konnten die Anwendungsbedingungen nach der Einführung der Direktdemokratie im Schnitt verbessert werden. Weil auch hier nur ein Teil der Länder aktiv voranging und die anderen den Trend mehr oder weniger verschliefen, hat sich die Schere zwischen den Vorreitern und Nachzüglern seither nochmals vergrößert.

\section{Die Anwendung der Direktdemokratie auf sich selbst führt zu Dauerstrei- tigkeiten um die rechtliche Zulässigkeit und politische Ausgestaltung der plebiszitären Verfahren.}

Die Ausgestaltung der Plebiszite in den Länderverfassungen hat verfassungsrechtlich und -politisch ungute Folgen. Einerseits gibt sie der Verfassungsgerichtsbarkeit ein übermäßiges Gewicht, die über die rechtliche Zulässigkeit oder Unzulässigkeit eines Volksbegehrens in letzter Konsequenz zu entscheiden hat. Andererseits provoziert sie einen politischen Wettlauf zwischen plebiszitfreundlichen und -restriktiven Kräften, die die Volksgesetzgebung zu einer potenziellen Dauerbaustelle machen. Statt im Rahmen der direktdemokratischen Verfahren über inhaltliche Politik zu streiten, wird die direkte Demokratie selbst zum Streitgegenstand.

Der Versuch, den Widerspruch zwischen dem weitreichenden Grundmodell der Volksgesetzgebung und deren faktischen Entwertung in der Praxis durch eine Erleichterung der Verfahren zu beseitigen, ist aus normativer Sicht verständlich. Er findet deshalb in der Staatsrechtslehre zunehmend Unterstützung, was sich allerdings noch nicht in der Verfassungsrechtsprechung niederschlägt - diese hält an ihrer defensiven Auslegung der Volksrechte überwiegend fest. Auch die Parteien können die erreichten Verbesserungen nur zu einem geringen Teil auf ihre Fahnen schreiben. Die Fortschritte verdanken sich vielmehr den Bürgern selbst, die die Volksgesetzgebung ,in eigener Sache“ genutzt haben, um Erleichterungen durchzusetzen.

Eine Auswertung der Verfahren bestätigt dies. Schlüsselt man die 266 Volksinitiativen, -petitionen und -begehren, die laut Auskunft von „Mehr Demokratie“ im Zeitraum 1946 bis 2008 lanciert wurden, nach Themengebieten auf, liegt der 
Bereich „Demokratie, Staatsorganisation und Innenpolitik“ mit 21 Prozent gleich an zweiter Stelle - nach „Bildung und Kultur“ mit 29 Prozent. ${ }^{26}$ Etwa die Hälfte der demokratiepolitischen Begehren entfallen wiederum allein auf die direkte Demokratie. Betrachtet man den Zeitraum seit 1991, ab dem die plebiszitären Elemente flächendeckend eingerichtet wurden bzw. Bestand hatten, liegt der Anteil sogar bei zwei Dritteln. ${ }^{27}$ Zwar gelang es nur in vier Fällen (einer in Bayern und drei in Hamburg), einen Volksentscheid zu erzwingen, von denen zwei - der Entscheid über die Einführung des kommunalen Bürgerentscheids in Bayern (1995) und der Entscheid über die Einführung des Bürgerentscheids in den Hamburger Bezirken (1998) - erfolgreich waren. Die erfolgreiche Selbstanwendung der Direktdemokratie lässt sich mit diesen Zahlen aber nicht annähernd erfassen. Denn auch wo die Vorstöße über das Initiativ- oder Begehrensstadium nicht hinauskamen, wurden sie von den Parlamenten in der Regel aufgegriffen. Auf diese Weise konnten z.B. in Berlin, Bremen und Thüringen teilweise erhebliche Verbesserungen erreicht werden. ${ }^{28}$

Die rasche Folge der mithilfe des Plebiszits herbeigeführten Änderungen rührt einerseits daher, dass viele Initiativen sich nicht auf die förmliche Verfassung bezogen, wo die Änderungshürden sehr hoch liegen, sondern auf die leichter veränderbaren Ausführungsgesetze. Zum anderen waren ihnen oftmals Versuche der Verfassungsgerichte oder des Gesetzgebers vorausgegangen, den erreichten Zustand zurückzudrehen und die Anwendbarkeit der direktdemokratischen Verfahren zu beschneiden. Dass die pro-plebiszitären Kräfte in diesem Hase-IgelRennen am Ende meistens die Nase vorn hatten, ist nicht verwunderlich und im Sinne einer Auflösung des oben dargestellten Widerspruchs zu begrüßen. Zur Idee der Verfassung gehört allerdings zugleich, dass sie auf eine gewisse Dauerhaftigkeit hin angelegt ist. Ständiger Streit um einen wichtigen Teil seiner konstitutionellen Grundlagen tut keinem Gemeinwesen gut. Das Hin und Her zwischen freundlicher und restriktiver Ausgestaltung, das die Volksgesetzgebung

26 Mehr Demokratie, Volksbegehrensbericht (Fn. 22), S. 12 f.

27 Rux, Direkte Demokratie (Fn. 21), S. $931 \mathrm{ff}$.

28 Besonders spektakulär war die Wirkung der beiden ,unecht“ (das heißt lediglich am Zustimmungsquorum) gescheiterten Initiativen für erleichterte Volksbegehren und -entscheide in Hamburg (1998 und 2007), denen eine klare Mehrheit der Wähler zugestimmt hatte. Dies wurde von den Regierenden als Aufforderung verstanden, die Volksgesetzgebung grundlegend zu reformieren. Dass das ehemalige Schlusslicht Hamburg heute unter allen Bundesländern die anwenderfreundlichsten Verfahren hat, verdankt sich also ganz unmittelbar der direkten Demokratie. 
induziert, kann deshalb auch die Befürworter nicht kalt lassen, weil es der Akzeptanz der Direktdemokratie langfristig schadet. ${ }^{29}$

\section{Die Integration der Volksgesetzgebung wird auf der Bundesebene durch die Mitwirkungsrechte der Länder im Gesetzgebungsprozess zusätzlich er- schwert.}

Vor dem Hintergrund der Verfassungslage in den Ländern ist es wenig erstaunlich, dass sich auch die Diskussion um die Einführung von Plebisziten in das Grundgesetz weitgehend auf die Volksgesetzgebung kapriziert. Entsprechend sehen die Mehrzahl der Vorschläge vor, das Modell, wie es in Ländern und Kommunen existiert, für den Bund einfach zu übernehmen. Die Volksgesetzgebung ist sozusagen ,gesetzt“" und wird - auch im Lichte möglicher Alternativen - nicht weiter hinterfragt. Die Befürworter einer plebiszitären Verfassungsergänzung richten ihr Augenmerk deshalb ausschließlich auf die Anwendbarkeit der direktdemokratischen Verfahren - ganz so wie in den Ländern.

Symptomatisch dafür ist der von der rot-grünen Regierung 2002 eingebrachte Gesetzentwurf für eine Einfügung direktdemokratischer Elemente ins Grundgesetz, der sich nicht nur exakt am selben Modell der Volksgesetzgebung orientierte (unter Ausschluss aller anderen denkbaren Varianten), sondern für diese zugleich weniger Ausschlussgegenstände und niedrigere Hürden vorsah, als sie in den Ländern zu der Zeit im Durchschnitt bestanden. Mit Blick auf die zusätzlichen Schwierigkeiten, die eine Einführung der Volksgesetzgebung auf Bundesebene aufwerfen würde, war das nicht sehr glaubwürdig. Diese ergeben sich zum einen aus der föderativen Aufgabenverteilung, die die Verantwortung für die wichtigsten Politikbereiche (mit Ausnahme von Schule und Hochschule) dem Bund zuweist - hier dürfte sich eher eine zurückhaltendere Ausgestaltung der Volksrechte empfehlen. Zum anderen - und damit verbunden - muss geklärt werden, wie die Zweite Kammer im Volksgesetzgebungsverfahren zu beteiligen ist. Dieses Problem entfällt sowohl auf der einzelstaatlichen Ebene, wo in der Bundesrepublik nach der durch Volksbegehren erzwungenen Abschaffung des

29 Auch in Hamburg wird der 2009 gefundene Verfassungskompromiss wieder in Frage gestellt. Nach dem erfolgreichen Volksbegehren gegen die Schulreform mehren sich einerseits die Stimmen, die die Zugänglichkeit der Verfahren durch höhere Quoren erschweren wollen (so der frühere Bürgermeister Henning Voscherau); zum anderen wird die verfassungsrechtliche Zulässigkeit des doppelten Ja beim Volksentscheid angezweifelt, wenn neben dem Volksbegehren eine Konkurrenzvorlage der Bürgerschaft mit zur Abstimmung steht. 
bayerischen Senats heute keine Zweite Kammer mehr existiert, als auch in den meisten anderen nationalen politischen Systemen, in denen die Zweiten Kammern zumeist nur über abgestufte Beteiligungsrechte (in Gestalt eines suspensiven Vetos) verfügen. In Deutschland wirkt der Bundesrat demgegenüber im überwiegenden Teil der Gesetzgebung gleichberechtigt mit. Umso bemerkenswerter ist, dass die sich daraus mit Blick auf die Plebiszite ergebenden Konsequenzen erst in jüngster Zeit zum Gegenstand rechts- und politikwissenschaftlicher Untersuchungen geworden sind. ${ }^{30}$

Der normative Ausgangspunkt ist einfach: Dem Volk die Möglichkeit einzuräumen, selbst als Gesetzgeber in Aktion zu treten, ergibt offenkundig nur dann Sinn, wenn es beim abschließenden Gesetzesbeschluss die staatlichen Organe verdrängen, das heißt: sich vollständig an deren Stelle setzen kann. ${ }^{31}$ Ein Zustimmungsrecht des Bundesrates oder einer anders gearteten repräsentativen Körperschaft würde dem zuwiderlaufen. Außerdem wäre sie mit der heutigen Unterscheidung von Zustimmungs- und Einspruchsgesetzen nicht vereinbar. Die Kategorie der Einspruchsgesetze müsste im Grunde entfallen, da das Volk nach einem Einspruch des Bundesrates ansonsten gezwungen wäre, gleich nochmal abzustimmen. Dasselbe gilt für die Anrufung des Vermittlungsausschusses und das anschließende Vermittlungsverfahren, die nur durch gewählte oder delegierte Vertreter wahrgenommen werden können und deshalb ebenfalls keinen Bestand mehr hätten. Dabei zählen gerade sie zu den funktional unentbehrlichen Bestandteilen der föderativen Mitregierung.

Ein Zustimmungsrecht des Bundesrates zum anschließenden Gesetzesbeschluss halten heute nur noch wenige Autoren für zwingend geboten. Die meisten Verfassungsrechtler vertreten die Ansicht, dass das Ewigkeitsgebot des Art. 79 Abs. 3 lediglich die ,grundsätzliche Mitwirkung der Länder bei der Gesetzgebung" schütze und keine Aussage darüber treffe, in welcher Form diese Mitwirkung zu erfolgen habe. Positiv gewendet heißt das, dass die Mitwirkung auch durch die „Landesvölker“ selbst erfolgen kann. Das einschlägige Beispiel liefert die Schweiz, wo bei einem Verfassungsreferendum nicht nur die Mehrheit des Volkes zustimmen muss (,Volksmehr"), sondern auch die Mehrheit der Kan-

30 O. Jung, Direkte Demokratie und Föderalismus. Die grundsätzliche Mitwirkung der Länder bei der Volksgesetzgebung im Bund, in: S. Brink/H. A. Wolff (Hrsg.), Gemeinwohl und Verantwortung. Festschrift für Hans Herbert von Arnim zum 65. Geburtstag, 2004, S. 353 ff.; D. Estel, Bundesstaatsprinzip und direkte Demokratie im Grundgesetz, 2006; S. Blasche, Die Mitwirkung der Länder an der Gesetzgebung. Eine verfassungsdogmatische Untersuchung zu Art. 79 Abs. 3, 2. Var. GG vor dem Hintergrund einer möglichen Einführung von Volksgesetzgebung in das Grundgesetz, 2006.

31 H. Maurer, Plebiszitäre Elemente in der repräsentativen Demokratie, 1997, S. 27. 
tonsvölker („Ständemehr“; vgl. Art. 140 f., 142 Abs. 1 u. 2 BV). Dem Bürger steht dabei lediglich eine Stimme zu, die gleichzeitig als seine Volks- und Kantonsstimme gewertet wird. ${ }^{32}$

Die Übertragung dieses Modells auf die Bundesrepublik hätte den Vorteil, dass sie die unterschiedlichen Stimmengewichte der Länder im Bundesrat berücksichtigen könnte; die Mehrheit des Landesvolkes wäre dann gleichbedeutend mit der Abgabe der Bundesratsstimmen des Landes. Dies erschien den Befürwortern der direkten Demokratie seinerzeit so plausibel, dass sie die Ländermehr-Lösung 1993 in ihrem Vorschlag für eine plebiszitäre Ergänzung des Grundgesetzes verankerten, der anschließend der Gemeinsamen Verfassungskommission vorgelegt wurde. Als die rot-grüne Koalition 2002 ihren eigenen Entwurf auf den Weg brachte, konnte sie an dieses Konzept anknüpfen.

Die Schwächen der Ländermehr-Lösung erschließen sich erst auf den zweiten Blick. Folgt man der Argumentation von Estel ${ }^{33}$, muss ihre Übernahme in der Bundesrepublik aus zwei Gründen scheitern. Zum einen verkörpere die Schweiz ein ganz anderes Föderalismusmodell, bei dem die an einem Volksentscheid teilnehmenden Bürger nicht als einheitliches Bundesvolk, sondern als Zusammenschluss der Kantonsvölker aufträten, wohingegen in der stärker unitarisch geprägten Bundesrepublik die fiktive Trennung von Bundes- und Landeszugehörigkeit keinen Sinn ergebe. Zum anderen könne das Ländermehr im deutschen Fall keinen Ersatz für ein föderatives Vertretungsorgan bieten, weil nur dieses funktional in der Lage sei, die Länderinteressen wahrzunehmen. Der Unterschied zur Schweiz folgt hier aus der Zuständigkeitsverteilung zwischen beiden Ebenen. In der Schweiz verfügen die Kantone sowohl in der materiellen Gesetzgebung als auch bei der Umsetzung der Bundesgesetze über weitreichende eigene Befugnisse, was die Schutzfunktion einer föderativ konstituierten Zweiten Kammer bis zu einem gewissen Grade entbehrlich macht und die Wahrnehmung der Kantonsinteressen durch das Ständemehr als ausreichend erscheinen lässt. In der Bundesrepublik kommt man demgegenüber nicht umhin, den Ländern auch ein inhaltliches Mitgestaltungsrecht an den Bundesgesetzen einzuräumen, weil der Bund hier durch die Regelung der Verwaltungsverfahren, die Steuergesetzgebung und die Überwälzung von Finanzierungslasten unmittelbar in deren Domäne eingreift. Die Regelzuständigkeit der Länder für den Vollzug legitimiert on, in: ZParl 23 (1992), 166 ff.

33 Estel, Bundesstaatsprinzip (Fn. 30). 
dabei zugleich die im internationalen Vergleich unübliche Ausgestaltung der Zweiten Gesetzgebungskammer als Vertretungsorgan der Länderexekutiven. ${ }^{34}$

Gegen diese Argumentation ließe sich einwenden, dass das Gros der Gesetze lediglich Routineangelegenheiten umfasst, bei denen die Verwaltungskompetenz genauso gut auf anderem Wege - etwa durch eine gutachterliche Stellungnahme im Vorstadium des Volksentscheids - wahrgenommen werden könnte. Unter den wichtigen Gesetzen dürften sich andererseits viele Fälle finden, bei denen die Länderbelange häufig nur vorgeschoben sind und die Ablehnung durch den Bundesrat in Wahrheit aus parteipolitischen Gründen erfolgt. So vertretbar eine Umgehung der Länderkammer unter solchen Bedingungen scheint, scheidet sie als Lösung dennoch aus. Der Regierungsseite wäre damit ja die Möglichkeit gegeben, ihre Gesetzesvorhaben von vornherein auf dem plebiszitären Wege zu betreiben, um die gegnerische Mehrheit im Bundesrat auszuschalten. Die Zweckentfremdung eines verfassungsmäßigen Instruments würde hier gewissermaßen durch die Zweckentfremdung eines anderen Instruments beantwortet. Stellt man diese systemischen Weiterungen in Rechnung, so bleibt von der scheinbaren Eleganz des Schweizer Modells am Ende nicht mehr viel übrig.

\section{Ein systemgerechtes Modell der Direktdemokratie müsste anstelle der Volksgesetzgebung eine Kombination von Vetoinitiative und einfachem Re- ferendum vorsehen.}

Welche Konsequenzen ergeben sich aus der Analyse für die Weiterentwicklung der direkten Demokratie in den Ländern und auf Bundesebene? Der einfachste Weg, den Schwierigkeiten der systemischen Integration zu entrinnen, wäre die Abkehr vom Volksgesetzgebungsmodell. Für die Länderebene kann eine solche Lösung nach den Regeln der Pfadabhängigkeit mit ziemlicher Sicherheit ausgeschlossen werden. Nicht nur, dass die Abschaffung der bestehenden Volksrechte als demokratischer Rückschritt empfunden würde, der gegen die Mehrheitsmeinung der Bevölkerung kaum durchsetzbar wäre. Auch die Restriktionen des Modells weisen entwicklungsgesetzlich eher in Richtung einer (das Modell bekräftigenden) Verfahrenserleichterung. Es ist also davon auszugehen, dass es in den Ländern zu einer weiteren Verbesserung der Anwendungsbedingungen kommen wird, durch die die bisherigen Schlusslichter in Sachen Direktdemokratie zu den

34 F. Decker, Regieren im „Parteienbundesstaat“. Zur Architektur der deutschen Politik, 2011, S. 242 ff. 
Vorreitern aufschließen. Bei den letztgenannten scheint das Potenzial für Verfahrenserleichterungen mittlerweile weitgehend ausgereizt. Wie das Hamburger Beispiel zeigt, besteht die Herausforderung hier in erster Linie darin, den verfassungsrechtlichen Ist-Zustand in der Praxis zu bewähren, was ebenfalls nicht ohne Konflikte abgehen dürfte.

Die Länder werden also mit den Folgen, die sich aus der potenziellen Systemunverträglichkeit der Volksgesetzgebung ergeben, irgendwie zurechtkommen müssen. Theoretisch wäre es zwar vorstellbar, dass die Volksgesetzgebung - wie in der Schweiz - zu einer Zurückdrängung des Regierungs-Oppositions-Dualismus und mithin zu einer konsensuellen Umgestaltung des Regierungssystems beiträgt. Der Wandel hin zu einer Konkordanzdemokratie erscheint aber unter den speziellen Bedingungen des deutschen Länderparlamentarismus nicht sehr wahrscheinlich. Zum einen könnten sich die konsensuellen Wirkungen nur einstellen, wenn die direkte Demokratie zugleich auf der Bundesebene eingeführt würde. Zum anderen ist die mehrheitsdemokratische Logik der Regierungssysteme auf der Länderebene eher stärker ausgeprägt als im Bund, wo die Mitregierung der Zweiten Kammer für einen gewissen Konsensdruck sorgt. Deshalb stellt sich die Frage, ob man die Angleichung der Funktionslogiken nicht durch einen radikalen institutionellen Schnitt herbeiführen könnte, indem man das gewaltenfusionierende parlamentarische System in den Ländern durch ein präsidentielles System ersetzt. Das Pferd würde gewissermaßen von hinten aufgezäumt. Statt die Volksgesetzgebung dem bestehenden parlamentarischen System einzupassen, was eine restriktive Ausgestaltung der Plebiszite nach sich zieht, würde die Regierungsform auf das bestehende System der Direktdemokratie zugeschnitten. Die Volksrechte bekämen auf diese Weise einen breiteren Spielraum, könnten sich innerhalb des repräsentativen Systems als alternativer Pfad der Gesetzgebung leichter zur Geltung bringen. ${ }^{35}$

Das Vorbild für eine solche Reform sind die Kommunen, wo der flächendeckende Übergang von der parlamentarischen zur präsidentiellen Regierungsform in den neunziger Jahren zeitgleich mit dem Ausbau der plebiszitären Elemente (Bürgerbegehren und Bürgerentscheid) erfolgt ist. Bemerkenswert daran ist, dass das Argument der besseren wechselseitigen Verträglichkeit in diesem Prozess gar keine Rolle spielte. Die Direktwahl der Bürgermeister und die Stärkung der Bürgerrechte sollten unabhängig voneinander zu einer Stärkung der kommunalen Demokratie beitragen. Auch auf der Länderebene, wo der Vorschlag eines Sys-

35 F. Decker, Zwischen Placebo und Erfolgsmodell. Direkte Demokratie auf der Landesebene, in: ZParl 41 (2010), 564 (576 f.). 
temwechsels zum Präsidentialismus schon auf eine längere Tradition zurückblickt, wurde der Zusammenhang zur Volksgesetzgebung in der Literatur bisher nicht hergestellt. ${ }^{36}$

Abb. 1: Direkte Demokratie versus Regierungsform

\begin{tabular}{l|ccc}
\hline Ebene & Regierungsform & Direkte Demokratie & Verträglichkeit \\
\hline Bund & parlamentarisch & - & ja \\
Länder & parlamentarisch & Volksgesetzgebung & nein \\
\hline \multirow{2}{*}{ Kommunen } & quasi-präsidentiell & $\begin{array}{c}\text { Bürgerbegehren/ } \\
\text {-entscheid }\end{array}$ & ja \\
\hline
\end{tabular}

Auf der Bundesebene, wo ein solcher Systemwechsel ebenso wenig in Betracht kommt wie eine Abschaffung oder Entmachtung der Zweiten Kammer, kann die Empfehlung dagegen nur lauten, auf die Einführung einer (positiven) Gesetzesinitiative nach dem Modell der Länderverfassungen zu verzichten! Damit würde einerseits der Zwang entfallen, die Volksrechte in der konkreten Ausgestaltung durch Ausschlussgegenstände und Quoren zu beschränken, der einen permanenten verfassungsrechtlichen und -politischen Konfliktherd eröffnet; zum anderen bräuchte man sich nicht den Kopf zu zerbrechen, wie der Bundesrat in ein Volksgesetzgebungsverfahren einzubeziehen sei. Plebiszitäre Elemente auf der Bundesebene sind also nur dann systemverträglich, wenn sie das Prä der parlamentarischen Repräsentation erhalten. Ein anwendbares und in seinen institutionellen Rückwirkungen nutzbringendes Konzept könnte folgende Varianten umfassen:

1.) Konsultative Gesetzesinitiative. Mit ihr könnte der Gesetzgeber aufgefordert werden, sich mit einer bestimmten Thematik zu befassen. Eine rechtliche Verpflichtung, der Anregung zu folgen, besteht nicht, von der aber zweifellos eine starke politische Verpflichtungswirkung ausgehen würde. Ein wichtiger Anwendungsbereich wäre die Verfassungspolitik. Die konsultative Gesetzesinitiative könnte ein Korrektiv bilden, wenn eigene Machtinteressen der Parteien einer institutionellen Demokratisierung im Wege stehen. Sie würde also dort einspringen, wo die Volksgesetzgebung auf Länderebene heute ihre produktivsten Wirkungen entfaltet. 
2.) Vetoinitiative. Diese folgt dem Vorbild des Schweizerischen fakultativen Referendums. Sie eröffnet dem Volk die Möglichkeit, bereits beschlossene Gesetze einer nochmaligen Abstimmung zu unterwerfen und sie gegebenenfalls zu Fall zu bringen. Ausschlussgegenstände gibt es bis auf das Haushaltsgesetz keine. Die Hürden in der Eingangsphase müssten so bemessen sein, dass das Instrument nicht zu häufig zum Einsatz kommt. Hier könnte man sich an der Quote von fünf Prozent der Stimmberechtigten orientieren, die der rot-grüne Gesetzentwurf aus dem Jahre 2002 für die Einleitung eines Volksbegehrens vorgesehen hat.

3.) Einfaches Referendum. So wie dem Volk, so müsste man auch der Regierung die Möglichkeit einräumen, ihre eigenen Gesetze zur Abstimmung zu stellen, wenn regierungsinterne Blockaden und/oder oppositionelle Widerstände aus dem Bundesrat die reguläre Gesetzgebung erschweren. Eine verlorene Abstimmung könnte als Hebel dienen, um über eine vorzeitige Parlamentsauflösung Neuwahlen anzuberaumen. Dies würde in die Nähe eines plebiszitären Auflösungsrechts führen und wäre eine Möglichkeit, das verfassungsrechtlich strittige Verfahren nach Art. 68 des Grundgesetzes zu umgehen.

4.) Obligatorisches Referendum. Hierfür kommen in erster Linie internationale Verträge in Betracht, die die Übertragung von Souveränitätsrechten auf zwischenstaatliche Organisation regeln, wie z.B. der EU-Verfassungsvertrag, über den zehn der damals 25 Mitgliedstaaten durch Referendum entschieden haben. Der andere Anwendungsbereich sind Verfassungsrevisionen, bei denen man aber - um eine Überbeanspruchung zu vermeiden - eine sinnvolle Eingrenzung vornehmen müsste (in der Bundesrepublik etwa auf die Art. 1 bis 20 des Grundgesetzes).

VII. Die von einem Vetorecht des Volkes ausgehenden konsensuellen Wirkungen wären im bundesdeutschen Verfassungskontext zu begrüßen.

Mit Blick auf das oben behauptete Integrationsproblem der Initiative in ein gewaltenfusionierendes parlamentarisches System erscheint die Forderung nach einem plebiszitären Vetorecht auf den ersten Blick widersinnig. Dies gilt zumal insoweit, als die Volksgesetzgebung in der Verfassungspraxis der Länder der Wirkungsweise der Vetoinitiative sehr nahekommt. Warum sollte dann das eine Instrument systemunverträglich sein und das andere nicht?

Die Einführung der Vetoinitiative würde den Charakter des deutschen Regierungssystems nachhaltig verändern. Es ist eine oft wiederholte Binsenweisheit, dass das Veto seine Wirkung dabei nicht erst durch den tatsächlichen, sondern 
bereits durch den möglichen Gebrauch entfaltet. Als Handlungsoption in Reserve stehend, zwingt es die politischen Akteure, auf die referendumsfähigen Gruppen Rücksicht zu nehmen. Die Initiative sorgt also für eine breitere Interesseninklusion. Sie hält die Regierung an, etwaige Widerstände gegen ihre Gesetzesvorhaben schon im Vorwege einzukalkulieren und wirkt dadurch der Logik nach konsensuell.

Bei der Volksgesetzgebung werden die Konsenswirkungen durch institutionelle Querverbindungen zur parlamentarischen Gesetzgebung unterstützt. So können die Länderparlamente in der Bundesrepublik z.B. einen Volksentscheid abwenden, wenn sie sich dem Volksbegehren innerhalb einer bestimmten Frist ganz oder weitestgehend anschließen. Kommt es zum Volksentscheid, haben sie wiederum die Möglichkeit, einen eigenen Entwurf mit zur Abstimmung zu stellen. Auch dieser wird in der Regel das Anliegen der Initiative zumindest teilweise aufgreifen. Bei der Vetoinitiative muss der parlamentarische Gesetzgeber demgegenüber auf seine Einschätzung vertrauen, dass ein Vorhaben hinreichende Unterstützung findet. Die Überzeugungsarbeit bedarf entsprechend größerer Sorgfalt und setzt bereits in einem frühen Stadium des Gesetzgebungsprozesses ein. Weil das Monopol der politischen Gestaltung bei Parlament und Regierung verbleibt, greift die präventive Logik hier noch stärker als in einem Volksgesetzgebungsverfahren.

Umso mehr drängt sich die Frage auf, warum es sinnvoll sein soll, die Vetoinitiative ausgerechnet in ein parlamentarisches Regierungssystem einzuführen. Um sie zu beantworten, muss man sich vergegenwärtigen, dass nur die wenigsten Länder das gewaltenfusionierende Prinzip in Reinkultur kennen. Obwohl man es die Mutter aller westlichen Demokratien genannt hat, ist das britische Westminster-Modell, das in der Regierungsformenlehre als Prototyp der ,perfekten" Mehrheitsdemokratie gilt, unter den parlamentarischen Systemen die Ausnahme geblieben. Während Großbritannien seiner Regierung bis heute einen denkbar großen Handlungsspielraum einräumt, unterliegt dieser in den meisten anderen demokratischen Regierungssystemen mehr oder weniger engen verfassungsstaatlichen Grenzen. Statt von Parlamentssouveränität müsste man hier eher von Verfassungssouveränität sprechen. ${ }^{37}$ Letztere findet ihren Ausdruck in der Normenkontrolle durch eine unabhängige Verfassungsgerichtsbarkeit.

Die Begrenzung des mehrheitsdemokratischen Prinzips geht in den meisten parlamentarischen Systemen allerdings noch sehr viel weiter; sie erstreckt sich

37 H. Abromeit, Volkssouveränität, Parlamentssouveränität, Verfassungssouveränität: Drei Realmodelle der Legitimation staatlichen Handelns, in: PVS 36 (1995), 49 ff. 
auch auf die Repräsentationsebene, wo die Regierungen gezwungen sind, ihre legislative Gestaltungsmacht mit anderen Akteuren zu teilen. Von der Notwendigkeit der Koalitionsbildung über die vorsorgliche Einbindung wichtiger Interessengruppen bis hin zu den verbrieften Mitwirkungsrechten des Staatsoberhauptes oder einer föderativ konstituierten Zweiten Kammer lassen sich dabei verschiedene Varianten unterscheiden. Der niederländische Politikwissenschaftler Arend Lijphart ${ }^{38}$ hat für das Ensemble solcher machtteilenden Elemente den Begriff der Konsensdemokratie geprägt und diese dem mehrheitsdemokratischen Westminster-Parlamentarismus als Antipoden gegenübergestellt. Die mehrheitsdemokratischen Formen werden durch den Konsensualismus nicht vollständig verdrängt, sondern bleiben innerhalb der Institutionen wie auch im Verhältnis zwischen diesen weiter wirksam. Ihre faktische Suspendierung kann im Extremfall freilich soweit reichen, dass die parlamentarischen Verfahren nur noch als Fassade dienen, um die wahren Entscheidungswege zu verbergen.

An dieser Stelle kommen die plebiszitären Elemente ins Spiel. Als Korrektiv drängen sie sich vor allem dort auf, wo das parlamentarische Wechselspiel von Regierung und Opposition gestört oder ganz aufgehoben ist. Dies könnte einerseits der Fall sein, wenn eine bestimmte Partei oder eine Parteienkoalition über eine hegemoniale Position verfügt, die es ihr/ihnen erlaubt, ununterbrochen zu regieren. ${ }^{39}$ So geriet z.B. die Dominanz der CSU im Bundesland Bayern erträglicher, weil die direkte Demokratie den Wählern die Möglichkeit gab, der „Staatspartei“ ab und zu eine Niederlage beizubringen. Dass die Herrschaft der CSU damit letztlich zementiert wurde, unterstreicht die potenzielle Unverträglichkeit des plebiszitären Vetos mit dem alternierenden Regierungsprinzip. In ähnliche Richtung weist der italienische Fall. Hier diente die Einführung der - als abrogatives Referendum bezeichneten - Vetoinitiative im Jahre $1970^{40}$ der legitimatorischen Absicherung des Konkordanzsystems, das von der Democrazia Cristiana etabliert worden war, um die Kommunisten von der Macht dauerhaft

38 A. Lijphart, Patterns of Democracy, Government Forms and Performance in Thirty-Six Countries, New Haven/London 1999.

39 Auch in der Schweiz ist der Ausbau der Direktdemokratie vor diesem Hintergrund erfolgt. Nachdem der siebenköpfige Bundesrat nach der Bundesstaatsgründung $1848 \mathrm{zu}-$ nächst ausschließlich von Vertretern der Liberalen besetzt wurde, schuf die Einführung und Nutzung der Volksrechte die Voraussetzung dafür, dass die oppositionellen Parteien (Katholiken, Bauernpartei und Sozialdemokraten) nach und nach in die Regierung integriert werden konnten.

40 Genauer gesagt handelte es sich um den Erlass eines Ausführungsgesetzes - das Referendum selbst war in Art. 75 der Verfassung bereits vorgesehen, konnte bis zu diesem Zeitpunkt aber nicht aktiviert werden. Vgl. A. Capretti, Direkte Demokratie in Italien, in: H.K. Heußner/O. Jung (Hrsg.), Mehr direkte Demokratie wagen, 2. Aufl. 2009, S. 161 ff. 
fernzuhalten. Mit dem Übergang zu einer bipolar geprägten Wettbewerbsdemokratie sollte sich diese Funktion in den neunziger Jahren erübrigen.

Auch Große Koalitionen stellen, wo sie über längere Zeit amtieren, im Rahmen des parlamentarischen Systems ein widriges Regierungsformat dar, das ein plebiszitäres Gegengewicht geboten machen könnte. Für die Bundesrepublik gilt das sogar in besonderem Maße, weil hier bei einem starken zahlenmäßigen Übergewicht der Regierung nicht nur die parlamentarische Kontrolle durch die Opposition leidet, sondern auch das Verfassungsgericht und die Länderkammer als Vetospieler weitgehend ausfallen. Überlegungen, in einer solchen Situation verstärkt auf die Reservebefugnisse des Bundespräsidenten zurückzugreifen, sind in der letzten Legislaturperiode zu Recht auf wenig Gegenliebe gestoßen. Anlass war die zweifache Weigerung des damaligen Amtsinhabers Horst Köhler, Gesetze der Großen Koalition durch seine Unterschrift in Kraft zu setzen. ${ }^{41}$ Statt dem Staatsoberhaupt die Rolle eines Vetospielers im Regierungsprozess einzuräumen, worauf sich die Verfassunggeber im Parlamentarischen Rat aus guten Gründen nicht verstehen konnten, böten plebiszitäre Elemente hier allemal einen besseren Ersatz.

Dasselbe gilt aber bereits unter normalen Regierungsbedingungen (wenn sich die beiden Noch-Volksparteien als Regierung und Opposition gegenüberstehen). Anders als bei einer Großen Koalition kann die Opposition hier mithilfe des Verfassungsgerichts und/oder des Bundesrates in die Rolle eines Vetospielers hineinschlüpfen, der die Gesetzesvorhaben der Regierung nicht nur kritisch begleitet, sondern aktiv zu Fall bringt. Dies birgt die Gefahr, dass sich beide Organe von ihrer eigentlichen Zweckbestimmung entfernen. Während das Verfassungsgericht als Mit- und Nebengesetzgeber auftritt, schützt die Länderkammer föderative Interessen vor, wo es in Wirklichkeit um parteipolitische Interessen geht. Verfügt das Volk selber über ein Vetorecht, bräuchte die Opposition solche Umwege nicht mehr zu gehen. Ein plebiszitäres Veto hätte zudem den Vorteil, dass es größere Konsenswirkungen erzeugt. Unter dem Strich wäre die Politik damit weniger blockadeanfällig als heute.

Dies führt zu einem letzten, allgemeiner gehaltenen Argument. Direktdemokratische Verfahren stellen auch eine Antwort darauf dar, dass die Wahlen in den parlamentarischen Parteiendemokratien an legitimierender Kraft einbüßen. Einerseits wird die Entscheidung darüber, wer regiert, im Zuge der Pluralisierung der Parteiensysteme von den Wählern weg in die Hände der Parteien oder genauer: Parteiführungen verlagert, die die Koalitionen unter sich ausmachen. An- 
dererseits fühlen sich die Wähler bei ihrer Stimmabgabe heute immer weniger an soziologische oder ideologische Gewissheiten gebunden. Um das wechselbereiter werdende Publikum zu umwerben, lohnt es sich für die Parteien deshalb zunehmend (oder kann es sich lohnen), unhaltbare Versprechungen abzugeben, eine in Wahrheit nicht vorhandene Handlungsmacht vorzutäuschen oder populistische Stimmungsmache zu betreiben. In einem direktdemokratischen Verfahren würde das allein aufgrund der einzuhaltenden Fristen nicht funktionieren. Die Volksrechte könnten also zumindest in Teilbereichen dazu beitragen, die politische Auseinandersetzung zu versachlichen. Damit würden sie gerade jenen Tendenzen einer plebiszitären Transformation der Parteiendemokratie entgegenwirken, die man ihnen selbst am liebsten vorhält. 